Check for updates

Cite this: J. Mater. Chem. A, 2020, 8 18726

Received 18th June 2020

Accepted 17th August 2020

DOI: $10.1039 / \mathrm{d} 0 \mathrm{ta0} 0031 \mathrm{c}$

rsc.li/materials-a

\title{
Unraveling vertical inhomogeneity in vapour phase polymerized PEDOT:Tos films $\uparrow$
}

\author{
Shangzhi Chen, (D) $\ddagger^{a}$ Ioannis Petsagkourakis, $\ddagger^{a}$ Nicoletta Spampinato, ${ }^{b}$ \\ Chaoyang Kuang, ${ }^{c}$ Xianjie Liu, ${ }^{a}$ Robert Brooke, ${ }^{d}$ Evan S. H. Kang, $\S^{a}$ Mats Fahlman, ${ }^{a}$ \\ Xavier Crispin, (D) ${ }^{a}$ Eleni Pavlopoulou ${ }^{\star b}$ and Magnus P. Jonsson (iD ${ }^{* a}$
}

\begin{abstract}
The conducting polymer poly(3,4-ethylenedioxythiophene) (PEDOT) forms a promising alternative to conventional inorganic conductors, where deposition of thin films via vapour phase polymerization (VPP) has gained particular interest owing to high electrical conductivity within the plane of the film. The conductivity perpendicular to the film is typically much lower, which may be related not only to preferential alignment of PEDOT crystallites but also to vertical stratification across the film. In this study, we reveal non-linear vertical microstructural variations across VPP PEDOT:Tos thin films, as well as significant differences in doping level between the top and bottom surfaces. The results are consistent with a VPP mechanism based on diffusion-limited transport of polymerization precursors. Conducting polymer films with vertical inhomogeneity may find applications in gradient-index optics, functionally graded thermoelectrics, and optoelectronic devices requiring gradient doping.
\end{abstract}

\section{Introduction}

Conducting polymers are solution-processable and flexible organic conjugated materials that are important for applications ranging from photovoltaic cells ${ }^{1}$ and thermoelectric generators ${ }^{2}$ to electrochromic displays ${ }^{3}$ and organic thin film transistors. ${ }^{4}$ Vapour phase polymerization (VPP) of poly $(3,4-$ ethylenedioxythiophene) (PEDOT) forms a particularly promising route to obtain high-quality conducting polymer films. ${ }^{5-10}$ Important features revealed in VPP PEDOT, including semi-metallic properties ${ }^{11,12}$ and ultrahigh electrical conductivity, ${ }^{9,10}$ have attracted research interests for exploration of both fundamental physics and practical applications. As example, we recently demonstrated that nanostructures of VPP PEDOT could sustain plasmons and be used as switchable optical nanoantennas. ${ }^{13}$ Notably,

\footnotetext{
${ }^{a}$ Laboratory of Organic Electronics, Department of Science and Technology (ITN), Linköping University, SE-60174 Norrköping, Sweden. E-mail: magnus.jonsson@liu.se ${ }^{b}$ Univ. Bordeaux, CNRS, Bordeaux INP, LCPO, UMR 5629, F-33600 Pessac, France. E-mail: eleni.pavlopoulou@enscbp.fr

${ }^{c}$ Department of Physics, Chemistry and Biology (IFM), Linköping University, SE-58183 Linköping, Sweden

${ }^{d}$ RISE Research Institutes of Sweden, Bio- and Organic Electronics, Bredgatan 35, SE60221 Norrköping, Sweden

$\dagger$ Electronic supplementary information (ESI) available. See DOI: $10.1039 /$ dota06031c

\$ These authors contributed equally.

$\S$ Current address: Department of Physics, Chungbuk National University, Cheongju 28644, Republic of Korea.
}

PEDOT films prepared by VPP typically possess strong anisotropy, manifested by significantly lower conductivity in the vertical direction across the film (out-of-plane). ${ }^{14,15}$ The out-of-plane behavior is crucial for many applications and therefore important to understand. Early studies on conducting polymers attributed anisotropy to a preferential orientation of the polymer crystallites. ${ }^{16,17}$ However, anisotropic behavior may also be related to stratification along the vertical direction of the film, which has only been rarely addressed. ${ }^{18,19}$

Here, we study stratification effects across thin films of VPP PEDOT doped with tosylate (PEDOT:Tos), which is a popular material for applications such as thermoelectrics, sensing, and electrochromics. ${ }^{20-22}$ Our study takes advantage of a collection of techniques, including grazing incidence wide-angle X-ray scattering (GIWAXS), X-ray photoelectron spectroscopy (XPS) and ultraviolet photoelectron spectroscopy (UPS). We found that microstructural anisotropy and vertical gradients in microstructure and doping level co-exist in the VPP PEDOT:Tos films. The origin for such inhomogeneities is consistent with a polymerization mechanism based on diffusion-limited transport of precursors. . $, 6,23$ We believe this study provides deeper understanding of the VPP process, which can be useful in further optimization of the properties of conducting polymers. In addition, the observed stratified properties across the PEDOT:Tos films may find use in specific applications, including anti-reflection coatings, tunable photodetectors, functionally graded thermoelectrics and gradient-index optics. ${ }^{24-26}$ 


\section{Experimental}

\subsection{Materials}

Clevios $^{\mathrm{TM}}$ C-B 54 V3 (54\% wt/wt iron(III) $p$-toluene sulfonate (Fe(III)-(Tos) $)_{3}$ ) in $n$-butanol) was purchased from Heraeus (Germany). EDOT monomer (142.18 $\left.\mathrm{g} \mathrm{mol}^{-1}\right)$ and the poly(ethylene glycol)-block-poly(propylene glycol)-block-poly(ethylene glycol) (PEG-PPG-PEG) (5800 $\mathrm{g} \mathrm{mol}^{-1}$ ) were purchased from Sigma-Aldrich. All materials were used as received with no further purification.

\subsection{Thin film preparation}

PEDOT:Tos films were prepared via vapor phase polymerization (VPP) of EDOT monomers in the presence of $\mathrm{Fe}(\mathrm{TOS})_{3}$ within a vacuum chamber. The vacuum chamber we used is a heated vacuo-temp vacuum desiccator (Selecta $\left.{ }^{\circledR}\right)$, instead of vacuum oven used by some groups. ${ }^{18}$ The oxidant solution was prepared by mixing $2 \mathrm{~g}$ of C-B $54 \mathrm{~V} 3,2 \mathrm{~g}$ of the tri-block co-polymer PEGPPG-PEG, and $5 \mathrm{~g}$ of ethanol (99.5 wt\%). In the oxidation solution, the concentrations for $\mathrm{Fe}(\mathrm{Tos})_{3}$ and PEG-PPG-PEG are $253 \mathrm{mM}$ and $46 \mathrm{mM}$, respectively. Films were deposited by spincoating the oxidant solution at $1500 \mathrm{rpm}$ for $30 \mathrm{~s}$ onto glass substrates. After $30 \mathrm{~s}$ baking on a hot plate of $70{ }^{\circ} \mathrm{C}$, the samples were transferred into the vacuum chamber ( 70 mbar, less than 1 minute of pumping was needed to reach this pressure). EDOT droplets were drop-casted onto glass substrates on a hot plate of $60{ }^{\circ} \mathrm{C}$ inside the chamber to ensure their evaporation. After 30 minutes of polymerization in the vacuum chamber, the samples were taken out from the chamber and baked on a hot plate at $70{ }^{\circ} \mathrm{C}$ for $2 \mathrm{~min}$. We note that the operating pressure may play a role in the VPP process (e.g. the evaporation of solvents in the oxidant). 70 mbar was selected based on the optimization of the VPP chamber in our lab. ${ }^{21,27}$ Then the samples were placed into a DI water bath. PEDOT:Tos films could be easily detached from the substrates during this step by applying small mechanical perturbation to the bath. The free-standing PEDOT:Tos film floating in the water bath could be manipulated by tweezers and re-deposited onto another substrates. The film can be turned upside down and transferred to the second substrate and we denote it as flipped sample for bottom surface characterization. The films without flipping or flipped by $360^{\circ}$ were noted as original sample. Both samples were washed by ethanol to remove the unreacted oxidants and monomers, followed by a nitrogen flow drying step.

\subsection{Optical, electrical and structural characterization}

Absorption spectra were studied using a UV-vis-NIR spectrometer PerkinElmer Lambda 900 in the range of 3300 to $400 \mathrm{~nm}$ with steps of $1 \mathrm{~nm}$. AFM topography and phase images were carried out using a Veeco Dimension 3100 with tapping mode. Sheet resistance of the film, $R_{\mathrm{s}}$, was measured directly on the film surface via a four-point probe set-up using a Signatone Pro4 S-302 resistivity stand and a Keithley 2400. Film thickness $t$ was measured by a surface profiler (Dektak 3st Veeco). The conductivity was calculated using the equation $\sigma=1 /\left(R_{\mathrm{S}} \times t\right)$. Contact angle measurements were conducted using a CAM optical contact angle and surface tension meter (KSV Instruments). $8 \mu \mathrm{L}$ droplets of de-ionized (DI) water, ethanol, and acetone were added on top of the spin-coated oxidant thin films via syringe.

\subsection{XPS and UPS}

VPP PEDOT:Tos thin films were prepared on ITO-coated glass substrates $(1 \mathrm{~cm} \times 1 \mathrm{~cm}, 90 \mathrm{~nm}$ ITO). Photoemission experiments were performed using a Scienta ESCA 200 spectrometer in an ultrahigh vacuum with a base pressure of $10^{-10} \mathrm{mbar}$. The measurement chamber was equipped with a monochromatic $\mathrm{Al}(\mathrm{K} \alpha) \mathrm{X}$-ray source providing photons with $1486.6 \mathrm{eV}$ for X-ray photoemission spectroscopy (XPS). The XPS experimental condition was set so that the full width at half maximum of the clean $\mathrm{Au} 4 \mathrm{f}^{7 / 2}$ line was $0.65 \mathrm{eV}$. All spectra were collected at room temperature with a photoelectron take off angle of $0^{\circ}$ (normal emission). Deconvolution of the $\mathrm{S}(2 \mathrm{p})$ core level spectra for PEDOT:Tos samples were carried out on the basis of a priori knowledge of the chemical composition. ${ }^{22,28}$ During the deconvolution of the $\mathrm{S}(2 \mathrm{p})$ spectra, the energy difference between the $S\left(2 p_{1 / 2}\right)$ and $S\left(2 p_{3 / 2}\right)$ spin-orbit components was kept at $1.2 \mathrm{eV}$, and the intensity ratio fixed at $1: 2$. Although XPS data fitting may include some uncertainty, the resulting differences in extracted ratios will be small and not show significant deviations. Ultraviolet Photoelectron Spectroscopy (UPS) experiments were carried out in a UHV surface analysis system equipped with a Scienta-200 hemispherical analyzer. The base pressure of a sample analysis chamber is $2 \times 10^{-8}$ mbar. UPS was performed using a standard He-discharge lamp with HeI $21.22 \mathrm{eV}$ as excitation source and an energy resolution of 50 meV. The work function of the films was extracted from the determination of the high binding energy cutoff of the UPS spectra by applying a bias of $-3 \mathrm{~V}$ to the sample.

\subsection{GIWAXS}

The internal microstructure of the VPP PEDOT:Tos thin films was characterized by Grazing Incidence Wide Angle X-ray Scattering (GIWAXS). VPP PEDOT:Tos films for GIWAXS were prepared on 1-inch silicon substrates with thermal oxide layers. GIWAXS measurements were performed on the Dutch-Belgian Beamline (DUBBLE CRG), station BM26B, at the European Synchrotron Radiation Facility (ESRF), Grenoble, France. ${ }^{29}$ The energy of the X-rays was $12.66 \mathrm{keV}$, and the sample-to-detector distance was set as $11.15 \mathrm{~cm}$. Zero-incidence angle is found by following a well-established sample alignment procedure that includes consecutive z-scans and pitch-scans. A high precision motor was used to control the angle of incidence. For smooth films like those studied herein, the error in the incident angle is less than $0.01^{\circ}$. Four angles of incidence, $\alpha_{i}$, were used to probe the samples with different penetration depths, namely $0.10^{\circ}$, $0.14^{\circ}, 0.16^{\circ}$, and $0.20^{\circ}$. The diffracted intensity was recorded by a Frelon CCD camera and was normalized by the incident photon flux and the acquisition time (30 s). Flat field, polarization, solid angle, and efficiency corrections were subsequently applied to the 2 D GIWAXS images. ${ }^{30}$ The scattering vector $q$ was defined with respect to the center of the incident beam and has 
a magnitude of $q=(4 \pi / \lambda) \sin (\theta)$, where $2 \theta$ is the scattering angle and $\lambda$ is the wavelength of the X-ray beam (0.98 $\AA$ ). Herein we present the wedge corrected images where $q_{\mathrm{r}}\left(q_{\mathrm{r}}^{2}=q_{x}{ }^{2}+q_{y}{ }^{2}\right)$ and $q_{z}$ are the in-plane and near out-of-the-plane scattering vectors, respectively. The scattering vectors are defined as follows:

$$
\left(\begin{array}{c}
q_{x} \\
q_{y} \\
q_{z}
\end{array}\right)=\left(\begin{array}{c}
(2 \pi / \lambda)\left(\cos \left(2 \theta_{\mathrm{f}}\right) \cos \left(\alpha_{\mathrm{f}}\right)-\cos \left(\alpha_{\mathrm{i}}\right)\right) \\
(2 \pi / \lambda)\left(\sin \left(2 \theta_{\mathrm{f}}\right) \cos \left(\alpha_{\mathrm{f}}\right)\right) \\
(2 \pi / \lambda)\left(\sin \left(\alpha_{\mathrm{f}}\right)+\sin \left(\alpha_{\mathrm{i}}\right)\right)
\end{array}\right)
$$

where $\alpha_{\mathrm{f}}$ is the exit angle in the vertical direction and $2 \theta_{\mathrm{f}}$ is the in-plane scattering angle, in agreement with standard GIWAXS notation. ${ }^{31}$ The $1 \mathrm{D}$ scattering patterns are presented herein after background subtraction. The polar plots were calculated on the background-subtracted $2 \mathrm{D}$ wedge-corrected images.

\section{Results \& discussion}

\subsection{Bulk properties of original and flipped PEDOT:Tos thin films}

We prepared PEDOT:Tos (chemical structure shown in Fig. 1c) thin films using the VPP method, as illustrated in Fig. 1a and detailed in the Experimental section. In brief, the method contains four main stages: (1) deposition of the oxidant film onto the substrate; (2) transferring the film into a heated vacuum chamber filled with EDOT monomers in vapour phase; (3) growth of PEDOT film on top of the oxidant by its exposure to EDOT; and (4) water bath washing to remove unreacted precursors and byproducts. In this work, we made films with thickness of about $150 \mathrm{~nm}$ and in-plane electrical conductivity of around $600 \mathrm{~S} \mathrm{~cm}^{-1}$, in the same order as previous studies. ${ }^{\mathbf{1 4}}$ Owing to delamination during the washing step, the films could be easily detached from the substrate and transferred to new substrates with either the front side up (original samples) or bottom side up (flipped samples), as indicated in Fig. 1 b.

UV-vis-NIR absorption spectroscopy of original and flipped samples (Fig. 2a) show almost overlapping spectra, confirming that both thickness and bulk properties of the thin films remain largely unchanged during the flipping process. This result agrees with the PEDOT:Tos not being dissolved in or reacting with either water or ethanol during the flipping process., Concerning surface morphology, height and phase images from atomic force microscopy (AFM) also do not show any observable differences between the two surfaces, with surface roughness of $14.3 \mathrm{~nm}$ and $15.6 \mathrm{~nm}$ for the top and bottom surfaces, respectively (Fig. 2b).

\subsection{Vertical inhomogeneity in VPP PEDOT:Tos thin films}

Both original and flipped PEDOT:Tos thin films were characterized by GIWAXS to extract structural information. We collected 2D scattering patterns for four different angles of incidence: $0.10^{\circ}, 0.14^{\circ}, 0.16^{\circ}$ and $0.20^{\circ}$. This approach allows retrieving information from different layers (or penetration depths) of the film. ${ }^{31-35}$ By increasing the angle of incidence, the $\mathrm{X}$-ray penetration depth increases and thus a thicker layer is probed. ${ }^{31-35}$ In the ESI, $\uparrow$ we provide calculations of estimated penetration depth with respect to the angle of incidence, for homogeneous PEDOT:Tos at the specific X-ray wavelength of the experiment. Starting from the top surface, at $0.10^{\circ}$, only a thin layer (less than $10 \mathrm{~nm}$ ) of the upper part of the film contributes to scattering. This probed layer becomes thicker for $0.14^{\circ}$ and $0.16^{\circ}$, while at $0.20^{\circ}$ the entire film is probed.

Fig. 3 presents the resulting 2D GIWAXS images after correction for the missing wedge. The corresponding background-subtracted 1D scattering patterns (intensity versus the scattering vector, $q$ ) are shown in the first row of Fig. S1. $\dagger$ The scattering features that are included in the data agree well

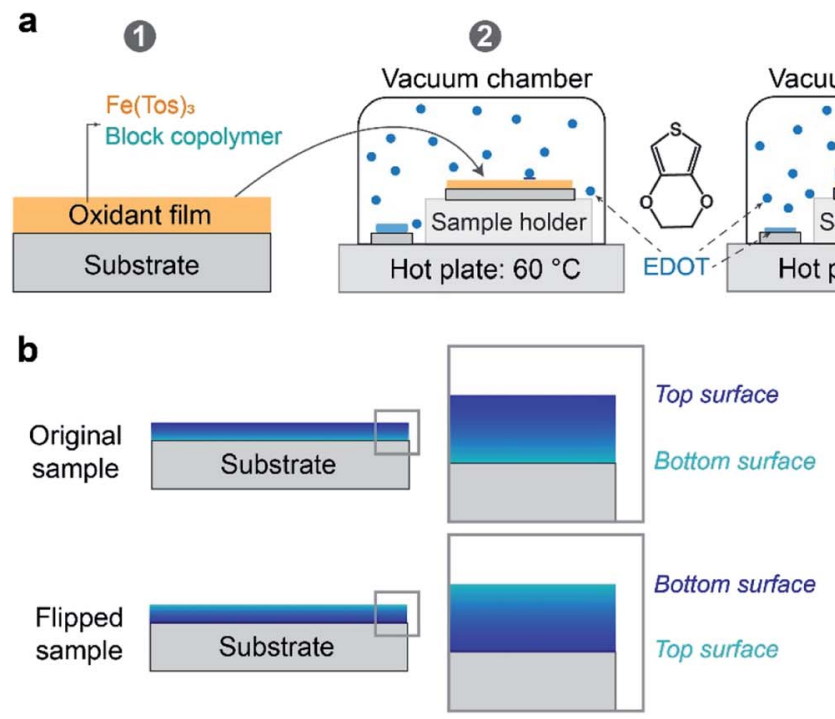

3 Vacuum chamber

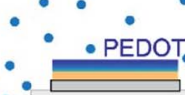

Sample holder

Hot plate: $60^{\circ} \mathrm{C}$

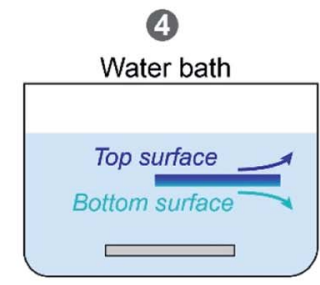

c

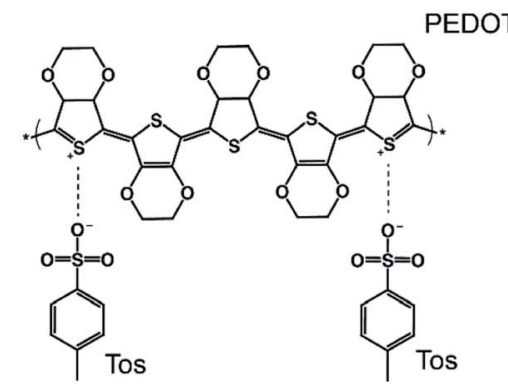

Fig. 1 (a) The process flow of VPP: spin-coating of oxidant film (1), monomer deposition (2), polymerization of monomers (3), and water bath cleaning (4). (b) The obtained thin film can be kept in its original orientation (top surface up) or turned over to its flipped orientation (bottom surface up) during the wash step. (c) Chemical structure of PEDOT:Tos. 
a

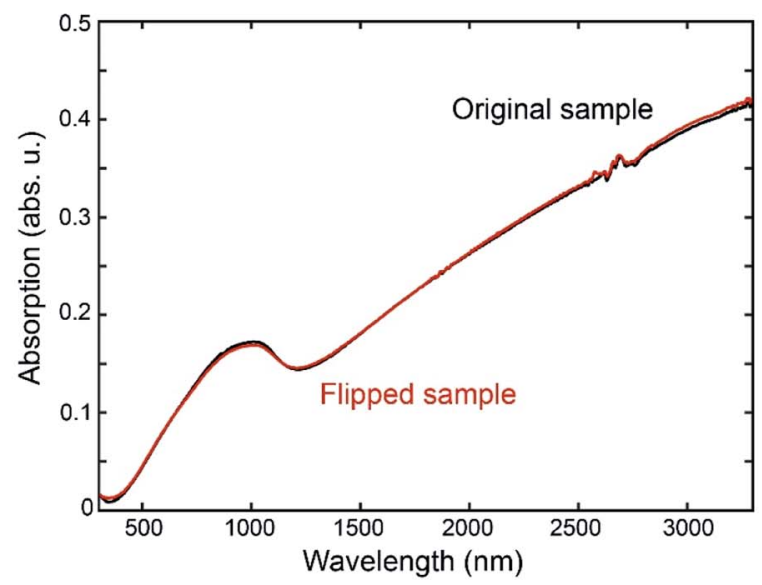

b

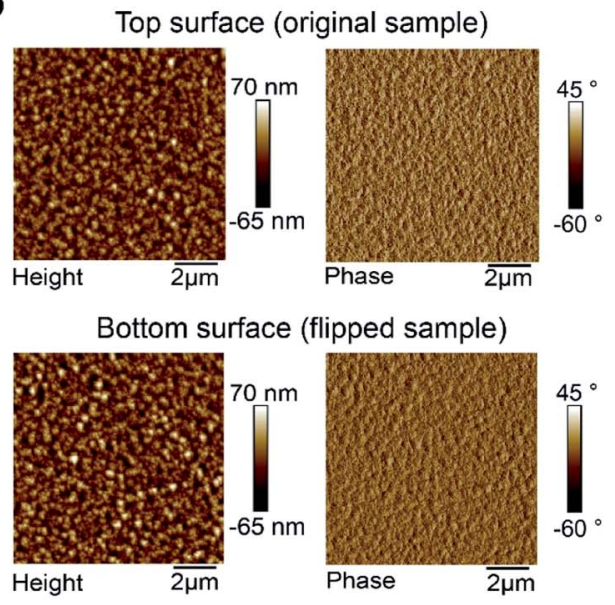

Fig. 2 (a) UV-vis-NIR absorption spectra of original and flipped PEDOT:Tos thin films. (b) AFM comparisons between the top (original sample) and bottom (flipped sample) surfaces: height images (left) and phase images (right). The average surface roughness for top and bottom surfaces are 14.3 and $15.6 \mathrm{~nm}$, respectively.

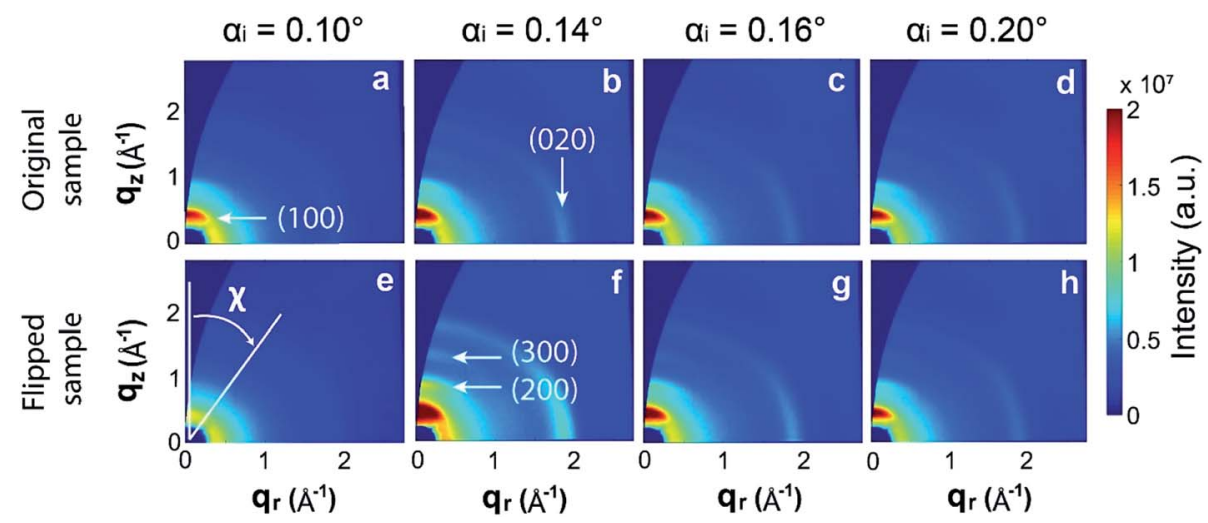

Fig. 3 GIWAXS 2D patterns (after wedge correction) collected for the original (top row) and flipped samples (bottom row), at four angles of incidence: $0.10^{\circ}, 0.14^{\circ}, 0.16^{\circ}$ and $0.20^{\circ}$. Polar angle $\chi$ and characteristic crystal planes are indicated in the figure.

with those reported for PEDOT:Tos. ${ }^{\mathbf{1 6}, 36}$ The bright spot at the out-of-plane direction, located at $0.44 \AA^{-1}$, is assigned to the (100) diffraction peak, followed in all cases by the second and third order reflections, (200) and (300). The scattering ring at around $1.86 \AA^{-1}$ is assigned to the (020) reflection.

Examining Fig. 3 and $\mathrm{S} 1 \uparrow$ reveals that both the intensity and polar distribution of the (100) spot varies with the angle of incidence. For both original and flipped samples, the scattered intensity reaches a maximum at the critical angle $\left(0.14^{\circ}\right)$ and then decreases at higher angles, i.e. at $0.16^{\circ}$ and $0.20^{\circ}$. These results are in accordance with the theory of grazing incidence scattering. ${ }^{31}$ Given the complexity of the grazing incidence diffuse X-ray scattering theory and the uncertainty on probed volumes at different angles, we avoid deriving other conclusions by comparing the intensities recorded for the same film at different angles. Instead, we focus the analysis on comparing the scattered intensities recorded at the same angle of incidence for the original and flipped films, for which the penetration depth and probed volume remain similar. At $0.10^{\circ}$, the integrated (100) peak intensity is lower for the flipped sample (i.e. when the bottom surface of the VPP PEDOT film is probed) than for the original sample. This suggests that the top surface is more crystalline compared with the bottom surface. Interestingly, we observe the opposite result at $0.14^{\circ}$ and $0.16^{\circ}$, where the (now thicker) probed layer appears to be more crystalline for the flipped sample compared with the original sample. At $0.20^{\circ}$, the scattering patterns are superposed, as expected. This angle is used for benchmarking, because the whole film contributes to scattering and, thus, the probing direction should not affect the results.

Already these observations demonstrate that the VPP films are not homogeneously structured along the vertical direction; the bottom layer that is first formed during VPP appears quite disordered while the layers that are subsequently formed above are much more crystalline, which explains why the relative integrated intensities between original and flipped films show a reverse relation when recorded at $0.14^{\circ}$. The slightly lower (100) integrated intensity of the original film at $0.16^{\circ}$ implies 
that the upper layer, despite being more crystalline than the bottom layer, is less crystalline than its underlying layers. Hence, the results indicate a non-monotonic variation of crystallinity across the VPP PEDOT:Tos.

Additional structural information can be derived by studying the orientation distribution function of the PEDOT:Tos crystallites. To do so, we integrate the (100) peak scattered intensity as a function of the polar angle $\chi$. The polar angle $\chi$ is defined with respect to the out-of-plane axis $q_{z}$, as depicted in Fig. 3e. These polar plots are presented in the second row of Fig. S1. $\dagger$ In the third row of Fig. $\mathrm{S} 1, \uparrow$ the polar plots are presented corrected for the amount of material oriented at each polar angle. Our films strongly resemble the case of in-plane powders, thus this correction is applied by multiplying the scattered intensity $(I)$ by $\sin (\chi) \cdot{ }^{36}$ These corrected $I \times \sin (\chi)$ versus $\chi$ plots correspond to the orientation distribution functions of the PEDOT:Tos crystallites. Given that we work with the (100) peak, crystallites oriented at $\chi<45^{\circ}$ are considered as edge-on oriented while those oriented at $\chi>45^{\circ}$ are considered as face-on. The constant non-zero background that remains in the intensity versus $\chi$ plots after background subtraction (second row of Fig. S1 $\dagger$ ) suggests that there is also a significant amount of isotropically oriented crystallites in the films. Fig. 4a shows schematic representations of edge-on, isotropic and face-on oriented crystallites.

The volume fractions of the differently orientated crystallites can be calculated by comparing the integrated areas of the corresponding features in the corrected $I \times \sin (\chi)$ plots (colored areas in Fig. 4d). Already the $I \times \sin (\chi)$ data presented in Fig. S1 $\uparrow$ show that the fraction of face-on crystallites in all cases is very small, while the fractions of edge-on and isotropic oriented crystallites are different for the original and the flipped films and vary with the angle of incidence. From the measurement at $0.10^{\circ}$ of the flipped sample, we learn that the bottom layer of the PEDOT:Tos film consists mainly of isotropic crystallites and less than 10\% edge-on crystallites (Fig. 4b). The result is different for the original sample, showing that the top surface contains around $25 \%$ edge-on crystallites (Fig. 4c). This difference between top and bottom surfaces is consistent with the higher disorder of the bottom surface discussed above based on the scattering patterns collected at $0.10^{\circ}$. Interestingly, increasing the angle of incidence (and thus the penetration
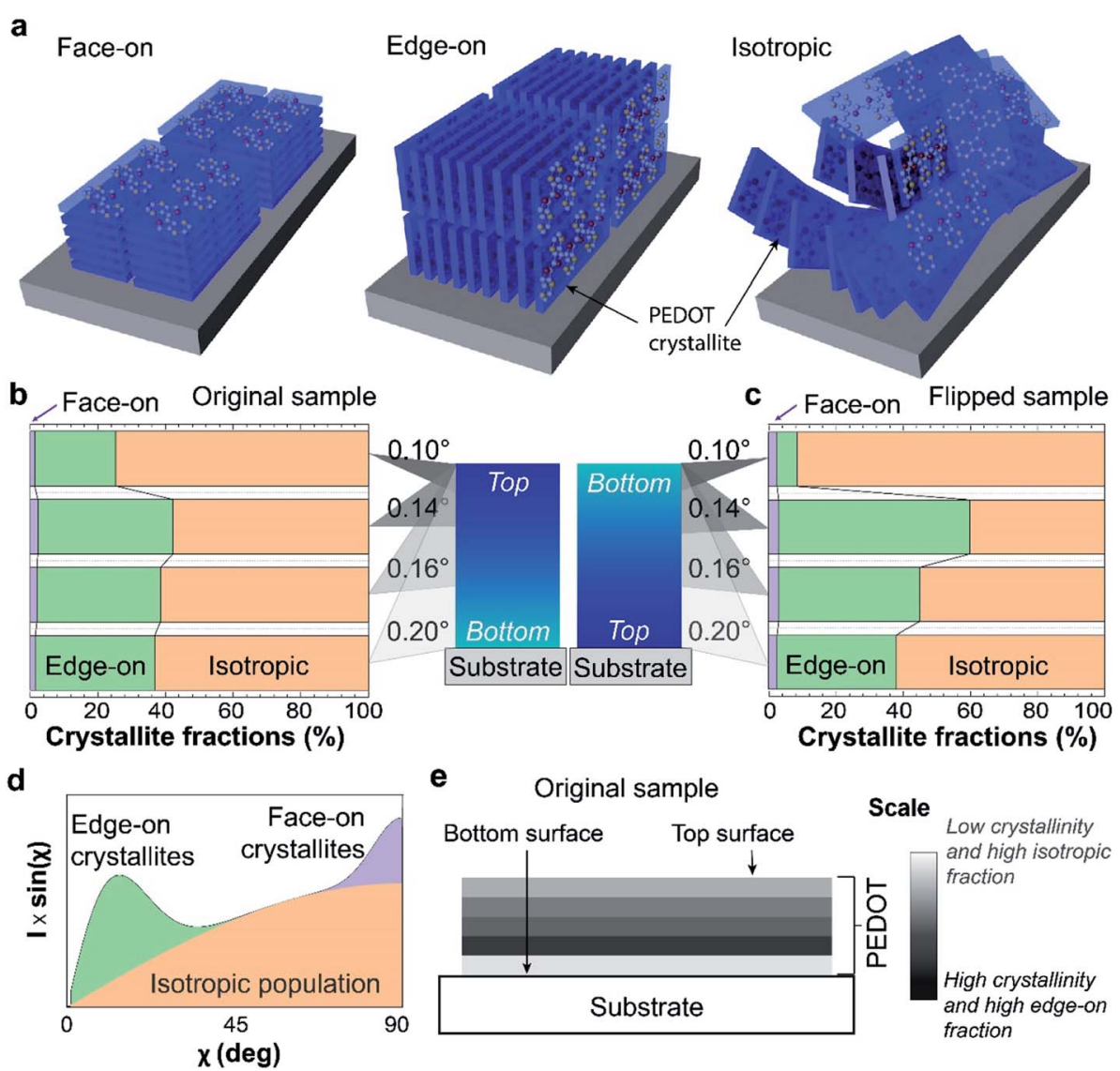

Fig. 4 (a) Schematic representations of face-on, edge-on, and isotropic orientation of the PEDOT:Tos crystallites with respect to the substrate. (b) and (c) The volume fractions of isotropic, edge-on and face-on oriented crystallites calculated for the various angles of incidence, for the original (top surface up) and flipped (bottom surface up) samples.(d) Example of a corrected polar plot, where the contributions of the various populations are depicted by the colored areas. (e) Schematic representation of the revealed stratification of the VPP PEDOT:Tos films. Darker zones correspond to layers with higher degree of order and edge-on fraction, while lighter zones correspond to layers with lower crystallinity and higher isotropic fractions as indicated by the scale bar. 
depth) to $0.14^{\circ}$ increased the measured fraction of edge-on crystallites for both original and flipped samples (to 60 and $40 \%$ for the flipped and original samples, respectively). Hence, both top and bottom surfaces consist of more isotropic crystallites than the corresponding regions further into the sample. At $0.16^{\circ}$ (probing a yet thicker part of the sample), the edge-on fraction decreases for both samples and at $0.20^{\circ}$ (probing the whole film), both samples show around 35\% fraction of edge-on crystallites. We underline that this comparison between orientation fractions for different angles of incidence is valid because the volume fractions are calculated internally, i.e. they are individually derived from the respective single polar plots.

These results suggest that the structural inhomogeneity along the vertical direction of the VPP PEDOT:Tos films is not only related to a variation of the relative degree of crystallinity (as discussed above), but also to a variation of the orientation of the PEDOT:Tos crystallites, namely a variation of the edge-on fraction. Combining all information derived by the two types of structural analyses allows us to make a general description of the stratification of the VPP PEDOT:Tos films. As depicted in Fig. 4e, the first bottom layer close to the growth substrate has low crystallinity and the PEDOT:Tos crystallites that are formed in that layer are mainly isotropically oriented. Above this bottom layer, a more crystalline layer is formed, in which the majority of crystallites is edge-on oriented. Subsequent layers formed on top are less crystalline than the second layer and exhibit a lower edge-on fraction, until arriving at the very top layer that is less crystalline than the layer underneath it. Yet, the very top layer is more crystalline and with a higher edge-on fraction compared with the first bottom layer of the film.

For conducting polymers, variations in microstructure often correlate with variations in charge carrier density (or doping level). ${ }^{11,37}$ To investigate this for our system, we examined top and bottom surfaces by XPS, which is a sensitive surface characterization technique (with penetration depth of about $10 \mathrm{~nm}$ ) for both chemical and electronic states of elements in materials. $^{38}$ The sulphur $\mathrm{S}(2 \mathrm{p})$ signals from the PEDOT backbone (166.5-162.5 eV, orange shaded areas) and Tos counterions (170-166 eV, green shaded area) can be separated due to different electronegativities of the surrounding atoms (see Fig. 5a and b). ${ }^{36,38,39}$ Because the Tos counterions are introduced to maintain electrical neutrality of the conducting polymer film, the concentration of counterions to monomers is indicative of the polymer's charge density, which we also refer to as oxidation level. The oxidation level or doping level of the samples can then be determined by the ratio of the integral areas of the low binding energy doublets $[S(2 p)$ from Tos] to the high binding energy doublets $[S(2 p)$ from PEDOT]. ${ }^{36,39}$ The results show that the bottom surface is highly doped, with an oxidation level of $32 \%$, or 1 Tos for 3 EDOT repeat units. By contrast, the top surface has only limited dopants, with an oxidation level of $22 \%$, or 1 Tos for around 5 EDOT repeat units. The observed difference in oxidation levels is not caused by the mild post-annealing ( 2 min at a temperature of $70{ }^{\circ} \mathrm{C}$ ), but remained also for nonannealed PEDOT:Tos (see Fig. $\mathrm{S} 2 \dagger$ ). These results suggest that the PEDOT:Tos films are vertically stratified also with respect to charge carrier density, while UPS (Fig. 5c) showed no clear difference in work functions $\left(\Phi_{\mathrm{f}}\right)$ between the bottom and top surfaces (4.68 eV for the top surface and $4.64 \mathrm{eV}$ for the bottom surface). The low binding energy region between $0 \mathrm{eV}$ and $3 \mathrm{eV}$ in the UPS results relates to the density of states of the $\pi$-electrons in the thiophene units of the PEDOT chains and its tail extends to the Fermi level. ${ }^{\mathbf{2 8 , 4 0}}$ The $\pi$-electrons of tosylate (as well as of polystyrene sulfonate ${ }^{\mathbf{4 1}}$ ), are localized on the first visible peak at $4 \mathrm{eV}$. Hence, the observed larger intensity at $4 \mathrm{eV}$ in the bottom surface is indicative of a higher density of tosylate compensating doping charge of PEDOT, i.e. a higher oxidation level (Fig. 5c inset). The variations in microstructural degree of order and doping level in the thin film may eventually result in vertical inhomogeneous electrical (e.g. charge density, mobility, and electrical conductivity) and thermoelectric (e.g. thermal conductivity, power factor) properties. The optical properties (e.g. refractive index or permittivity) of conducting polymers are strongly related to their charge density and mobility, as can be illustrated by modelling their complex and anisotropic refractive index using the Drude-Lorentz model. ${ }^{14}$ In turn, PEDOT layers with gradient refractive index properties could find applications as anti-reflection coatings. ${ }^{\mathbf{4 2 - 4 4}}$
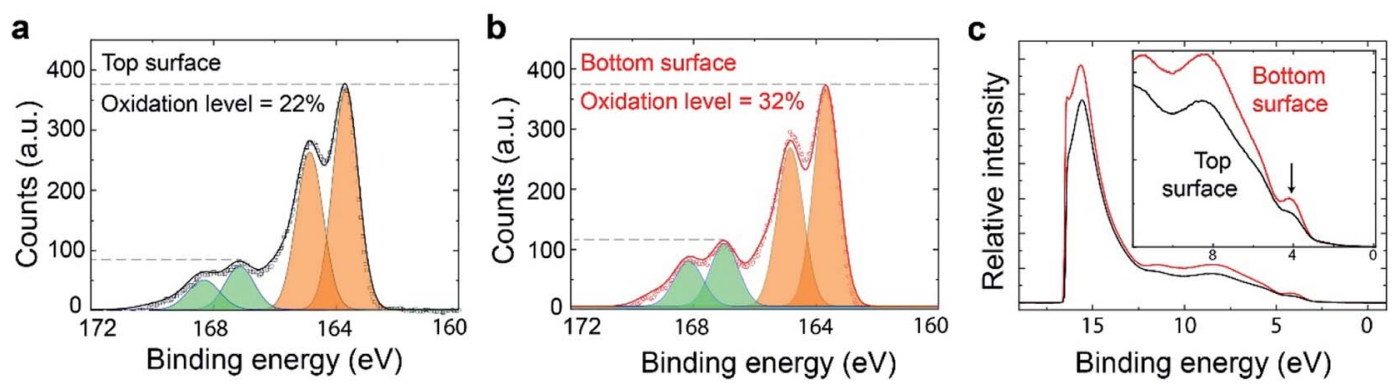

Fig. $5 \mathrm{~S}(2 \mathrm{p})$ XPS spectra (172-160 eV) for top (a) and bottom (b) PEDOT:Tos surfaces, showing different oxidation levels. The oxidation levels are calculated by the integral area ratio of two doublets from $\mathrm{S}(2 \mathrm{p})$ for the Tos counterion (shaded in green) and the PEDOT backbone (shaded in orange). Black squares and red dots are original data while black and red curves are fitted data for the oxidation level analysis. (c) UPS spectra of top (black) and bottom (red) surfaces. The inset shows an enlarged spectral region between 0 and $12 \mathrm{eV}$, to provide detailed information for the low binding energy range. The arrow in the figure indicates the difference between the two surfaces. 
a

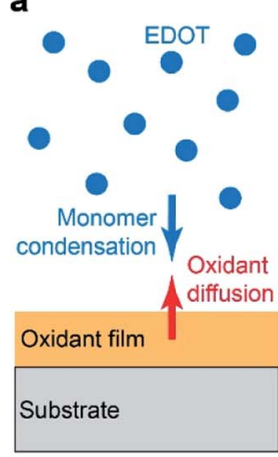

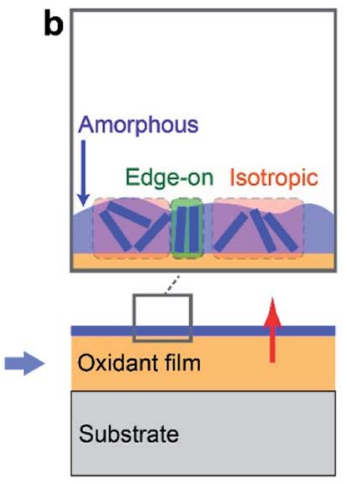

Stage 1: bottom

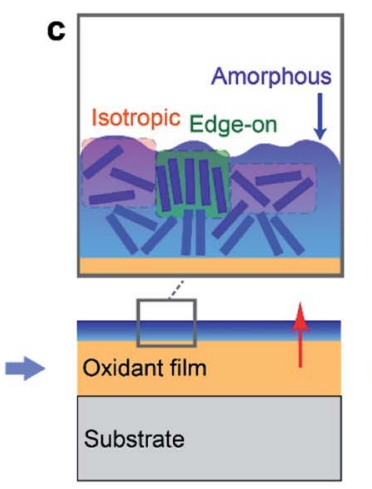

Stage 2: middle

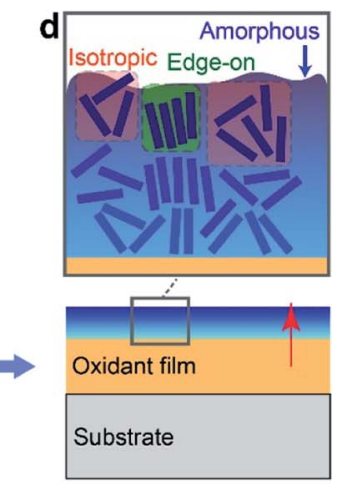

Stage 3: top

Fig. 6 Schematics of VPP PEDOT thin film growth mechanism: (a) two processes are indicated: monomer condensation (blue downwardpointing arrow) and oxidant diffusion (red upwards-pointing arrow). (b) Stage 1, polymerization of initial bottom layers: newly formed PEDOT are mostly amorphous with only a small fraction of crystallites (blue bricks) which are mainly isotropically oriented (shaded in red). (c) Stage 2, growth of middle layers: with the previous PEDOT layer as template/substrate, the crystalline fraction of PEDOT increases and edge-on orientation (shaded in green) becomes the main configuration. (d) Stage 3, growth of top layers: with the increase of film thickness, oxidant diffusion transport slows down and finally terminates the growth. Insufficient oxidant leads to incomplete polymerization and incomplete doping of PEDOT. The crystallinity decreases and more isotropic-oriented crystallites emerge. In (b-d), the shaded blue background behind the PEDOT crystalline bricks represents amorphous PEDOT regions.

\subsection{Diffusion-limited growth mechanism}

Based on the observed non-monotonic vertical inhomogeneity of the VPP PEDOT:Tos thin films, we turn to possible growth mechanisms. VPP growth of PEDOT is primarily an oxidative polymerization process where EDOT monomers are oxidized into cation radicals and finally polymerized with deprotonation. ${ }^{45}$ Therefore, the amount of EDOT monomers and oxidants (or precursors) that initiate the oxidation reaction (in this case: $\mathrm{Fe}(\mathrm{Tos})_{3}$ salts) are critical parameters for the rate of the reaction and quality of the produced films. The EDOT monomers are supplied in the form of vapour by mild heating of EDOT droplets while the oxidants are pre-spin-coated onto the growth substrate (see Fig. 6a). Therefore, two opposite processes can be defined: monomers condensing on the oxidant-containing surface (top down process) and oxidant molecules diffusing upwards to react with monomers (bottom up process ${ }^{23}$ ). While there is an excess of EDOT monomers, the availability of oxidant is limited due to two factors: (i) the total amount of oxidant in the film is limited and (ii) the bottom-up diffusion can be reduced and even blocked by already formed PEDOT layers. Hence, the amount of oxidant is likely to be the limiting factor for the reaction rate.

The local properties of each layer in the polymer film may be influenced by many factors, including the properties of the interface at which it was grown. In turn, previous research has shown that the vapor phase polymerization initially takes place at the air/oxidant interface, followed by growth at the interface between air and each newly formed polymer layer. ${ }^{23}$ Hence, the polymerization interface varies continuously during the growth process, which is in line with the observed inhomogeneities across the film. For example, crystallite orientation and morphology may depend on the crystallinity and surface energy of the underlying layer. ${ }^{46}$ While both the oxidant and the PEDOT:Tos films showed low contact angles, indicating hydrophilic behavior (see Fig. S3†), they differ significantly in terms of chemical nature, surface texture and also hardness. PEDOT has the tendency to crystallize and it is relatively hard compared with the soft and amorphous oxidant film, which due to the existence of the triblock polymer (PEG-PPG-PEG) acts as a self-healable gel. ${ }^{6}$ The different chemical nature between EDOT monomers and the oxidant molecules, as well as the disordered surface of the gel-like oxidant layer, can very well hinder the ordering of the initial PEDOT layer, which explains our finding that the initial PEDOT layer is more amorphous and with higher fraction of isotropic crystallites compared with subsequent layers (see Fig. 6b, stage 1). For polymerization of the next PEDOT layer, the initial bottom PEDOT layer serves as substrate. ${ }^{47}$ The growth surface is now of the same chemical nature as the formed layer, it is less soft than the oxidant layer and already contains some crystallites. The current growth substrate is thereby a template that favors the crystallization of PEDOT:Tos. This justifies our experimental finding concerning the higher crystalline volume fraction and more edge-on oriented crystallites of the intermediate layers (see Fig. 6c, stage 2). As the process continues, PEDOT increases in thickness and starts to act as a blocking layer that hinders diffusion of oxidant to the film/air interface, thereby affecting and finally terminating the growth after a certain thickness (see Fig. 6c, stage 3). The reduction in diffusion capability of the oxidant may also be related to an increase in viscosity due to evaporation of bound and trapped solvent molecules during film growth. ${ }^{6}$ The fact that there is a maximal thickness for VPP films ${ }^{39}$ agrees with the reduced diffusion transport of the oxidant. At stage 3 for the top layer formation, insufficient concentration of oxidant plays important roles. First, it may cause incomplete polymerization, incomplete chemical doping and produce shorter polymer chains, as implied by previous studies. ${ }^{27}$ Second, the local oxidant and corresponding 
counterion concentration can affect both the doping level (oxidation level) and microstructure of the conducting polymer. ${ }^{48,49}$ Density functional theory simulations have shown that $33 \%$ forms a 'magic number' for the oxidation level of PEDOT (equivalent to 1 counterion per 3 monomers), because such configuration is the most stable with lowest Gibbs free energy. ${ }^{\mathbf{5 0}}$ This value agrees well with that of the bottom interface of our VPP PEDOT:Tos films (32\%). The lower oxidation level of $22 \%$ at the top surface (see Fig. 5a and b) is consistent with insufficient transport of oxidant salt towards the top layer during the final polymerization stage. To sum up, a diffusion-limited oxidant transport mechanism can explain the observed non-monotonic vertical inhomogeneity in terms of variations in both structure and doping level for the VPP PEDOT:Tos samples. Based on our results, it may be possible to reduce the inhomogeneity effect via various pre- or post-treatments of the oxidant thin films, including modification of precursor recipes and substrate heating during polymerization. Indeed, VPP is a rather complex process and various factors may affect the film growth and final properties, such as detailed oxidant recipes, vacuum system setups, chamber pressure and temperature. Future work may study how the growth process is influenced by these and additional factors, including the type of oxidant and the size of counterions.

\section{Conclusions}

This report presents the investigation of vertical inhomogeneity across VPP PEDOT:Tos thin films. We found that both relative crystallinity and crystallite orientations varied non-monotonically across the thin film. Furthermore, the doping concentration was significantly lower $(22 \%)$ at the top surface compared with the bottom surface (32\%). These results are consistent with a diffusionlimited transport mechanism of the oxidant, which also agrees well with other related reported phenomena. Furthermore, we propose that the stratification across the PEDOT:Tos film is related to the continuously varying growth interface during polymer formation. The presented approach to investigate stratification effects in VPP PEDOT:Tos films may be applied also to other systems and improve understanding of different film formation processes that can potentially impact the performance of organic electronic devices. Conducting polymer films with known vertical property gradients may find use in various applications, including graded-index optics and graded thermoelectrics.

\section{Statement of contributions}

S. C., I. P., and R. B., E. S. H. K. prepared the films and performed electrical, optical and AFM characterizations. S. C. performed contact angle measurements. N. S. and E. P. performed GIWAXS measurements and analyzed the data. C. K., X. L., S. C, I. P, M. F., and X. C. performed XPS and UPS characterizations and analysis. S. C., I. P., E. P., and M. P. J. organized the data and wrote the manuscript. M. P. J. supervised the project. All the authors reviewed and commented on the manuscript.

\section{Conflicts of interest}

The authors declare no conflict of interest.

\section{Acknowledgements}

We thankfully acknowledge support from the Swedish Research Council, the Swedish Foundation for Strategic Research, the Wenner-Gren Foundations, the Knut and Alice Wallenberg Foundation, and the Swedish Government Strategic Research Area in Materials Science on Functional Materials at Linköping University (Faculty Grant SFO-Mat-LiU No. 2009 00971). The ESRF and the NWO are acknowledged for allocating beam time at the Dutch-Belgian beamline (DUBBLE) for the GIWAXS experiments.

\section{References}

1 G. Li, R. Zhu and Y. Yang, Nat. Photonics, 2012, 6, 153.

2 I. Petsagkourakis, N. Kim, K. Tybrandt, I. Zozoulenko and X. Crispin, Adv. Electron. Mater., 2019, 1800918.

3 A. A. Argun, A. Cirpan and J. R. Reynolds, Adv. Mater., 2003, 15, 1338-1341.

4 J. Rivnay, S. Inal, A. Salleo, R. M. Owens, M. Berggren and G. G. Malliaras, Nat. Rev. Mater., 2018, 3, 17086.

5 R. Brooke, P. Cottis, P. Talemi, M. Fabretto, P. Murphy and D. Evans, Prog. Mater. Sci., 2017, 86, 127-146.

6 D. Evans, M. Fabretto, M. Mueller, K. Zuber, R. Short and P. Murphy, J. Mater. Chem., 2012, 22, 14889-14895.

7 B. Winther-Jensen, D. W. Breiby and K. West, Synth. Met., 2005, 152, 1-4.

8 B. Winther-Jensen, J. Chen, K. West and G. Wallace, Macromolecules, 2004, 37, 5930-5935.

9 B. Cho, K. S. Park, J. Baek, H. S. Oh, Y.-E. Koo Lee and M. M. Sung, Nano Lett., 2014, 14, 3321-3327.

10 R. Brooke, J. F. Franco-Gonzalez, K. Wijeratne, E. Pavlopoulou, D. Galliani, X. Liu, R. Valiollahi, I. V. Zozoulenko and X. Crispin, J. Mater. Chem. A, 2018, 6, 21304-21312.

11 O. Bubnova, Z. U. Khan, H. Wang, S. Braun, D. R. Evans, M. Fabretto, P. Hojati-Talemi, D. Dagnelund, J.-B. Arlin and Y. H. Geerts, Nat. Mater., 2014, 13, 190.

12 S. Rudd, J. F. Franco-Gonzalez, S. Kumar Singh, Z. Ullah Khan, X. Crispin, J. W. Andreasen, I. Zozoulenko and D. Evans, J. Polym. Sci., Part B: Polym. Phys., 2018, 56, 97-104.

13 S. Chen, E. S. H. Kang, M. S. Chaharsoughi, V. Stanishev, P. Kühne, H. Sun, V. Darakchieva and M. P. Jonsson, 2019, arXiv:1907.11453.

14 S. Chen, P. Kühne, V. Stanishev, S. Knight, R. Brooke, I. Petsagkourakis, X. Crispin, M. Schubert, V. Darakchieva and M. P. Jonsson, J. Mater. Chem. C, 2019, 7, 4350-4362.

15 N. Kim, I. Petsagkourakis, S. Chen, M. Berggren, X. Crispin, M. P. Jonsson and I. Zozoulenko, Electric Transport Properties in PEDOT Thin Films, CRC Press Taylor \& Francis Group, 2019. 
16 K. E. Aasmundtveit, E. J. Samuelsen, L. A. A. Pettersson, O. Inganäs, T. Johansson and R. Feidenhans, Synth. Met., 1999, 101, 561-564.

17 H. Sirringhaus, P. J. Brown, R. H. Friend, M. M. Nielsen, K. Bechgaard, B. M. W. Langeveld-Voss, A. J. H. Spiering, R. A. J. Janssen, E. W. Meijer and P. Herwig, Nature, 1999, 401, 685.

18 A. Sharma, G. Andersson, J. Rivnay, J. F. Alvino, G. F. Metha, M. R. Andersson, K. Zuber and M. Fabretto, Adv. Mater. Interfaces, 2018, 5, 1800594.

19 B. J. Worfolk, S. C. Andrews, S. Park, J. Reinspach, N. Liu, M. F. Toney, S. C. B. Mannsfeld and Z. Bao, Proc. Natl. Acad. Sci., 2015, 112, 14138-14143.

20 S. Rudd, M. Dalton, P. Buss, A. Treijs, M. Portmann, N. Ktoris and D. Evans, Sci. Rep., 2017, 7, 1-6.

21 R. Brooke, J. Edberg, X. Crispin, M. Berggren, I. Engquist and M. P. Jonsson, Polymers, 2019, 11, 267.

22 O. Bubnova, Z. U. Khan, A. Malti, S. Braun, M. Fahlman, M. Berggren and X. Crispin, Nat. Mater., 2011, 10, 429.

23 R. Brooke, M. Fabretto, P. Hojati-Talemi, P. Murphy and D. Evans, Polymer, 2014, 55, 3458-3460.

24 M. WoŚKo, B. Paszkiewicz, T. Piasecki, A. Szyszka, R. Paszkiewicz and M. TŁAczaŁA, Opt. Appl., 2005, 35, 663667.

25 E. Mueller, Č. Drašar, J. Schilz and W. A. Kaysser, Mater. Sci. Eng. A, 2003, 362, 17-39.

26 H.-J. Seok, A. Ali, J. H. Seo, H. H. Lee, N.-E. Jung, Y. Yi and H.-K. Kim, Sci. Technol. Adv. Mater., 2019, 20, 389-400.

27 J. Edberg, D. Iandolo, R. Brooke, X. Liu, C. Musumeci, J. W. Andreasen, D. T. Simon, D. Evans, I. Engquist and M. Berggren, Adv. Funct. Mater., 2016, 26, 6950-6960.

28 X. Crispin, F. L. E. Jakobsson, A. Crispin, P. C. M. Grim, P. Andersson, A. Volodin, C. Van Haesendonck, M. Van der Auweraer, W. R. Salaneck and M. Berggren, Chem. Mater., 2006, 18, 4354-4360.

29 W. Bras, I. P. Dolbnya, D. Detollenaere, R. V. Tol, M. Malfois, G. N. Greaves, A. J. Ryan and E. Heeley, J. Appl. Crystallogr., 2003, 36, 791-794.

30 P. Muller Buschbaum, Adv. Mater., 2014, 26, 7692-7709.

31 G. Renaud, R. Lazzari and F. Leroy, Surf. Sci. Rep., 2009, 64, 255-380.

32 J. R. L. Parrill, P. Georgopoulos, Y. W. Chung and J. B. Cohen, J. Phys. IV, 1993, 3, C8-C411.
33 G. E. Stein, E. J. Kramer, X. Li and J. Wang, Macromolecules, 2007, 40, 2453-2460.

34 A. Vagias, Q. Chen, G. H. Ten Brink, D. Hermida-Merino, J. Scheerder and G. Portale, ACS Appl. Polym. Mater., 2019, 1, 2482-2494.

35 L. G. Parratt, Phys. Rev., 1954, 95, 359.

36 I. Petsagkourakis, E. Pavlopoulou, G. Portale, B. A. Kuropatwa, S. Dilhaire, G. Fleury and G. Hadziioannou, Sci. Rep., 2016, 6, 30501.

37 X. Wang, X. Zhang, L. Sun, D. Lee, S. Lee, M. Wang, J. Zhao, Y. Shao-Horn, M. Dincă and T. Palacios, Sci. Adv., 2018, 4, eaat5780.

38 K. Z. Xing, M. Fahlman, X. W. Chen, O. Inganäs and W. R. Salaneck, Synth. Met., 1997, 89, 161-165.

39 W. Shi, Q. Yao, S. Qu, H. Chen, T. Zhang and L. Chen, NPG Asia Mater., 2017, 9, e405.

40 W. A. Muñoz, X. Crispin, M. Fahlman and I. V. Zozoulenko, Macromol. Rapid Commun., 2018, 39, 1700533.

41 G. Greczynski, T. Kugler, M. Keil, W. Osikowicz, M. Fahlman and W. R. Salaneck, J. Electron Spectrosc. Relat. Phenom., 2001, 121, 1-17.

42 U. Schulz, F. Rickelt, H. Ludwig, P. Munzert and N. Kaiser, Opt. Mater. Express, 2015, 5, 1259-1265.

43 W. H. Southwell, Opt. Lett., 1983, 8, 584-586.

44 X. Li, J. Gao, L. Xue and Y. Han, Adv. Funct. Mater., 2010, 20, 259-265.

45 Y. H. Ha, N. Nikolov, S. K. Pollack, J. Mastrangelo, B. D. Martin and R. Shashidhar, Adv. Funct. Mater., 2004, 14, 615-622.

46 J. F. Franco-Gonzalez, N. Rolland and I. V. Zozoulenko, ACS Appl. Mater. Interfaces, 2018, 10, 29115-29126.

47 C. Duc, A. Vlandas, G. G. Malliaras and V. Senez, Soft Matter, 2016, 12, 5146-5153.

48 E. M. Thomas, M. A. Brady, H. Nakayama, B. C. Popere, R. A. Segalman and M. L. Chabinyc, Adv. Funct. Mater., 2018, 28, 1803687.

49 S. N. Patel, A. M. Glaudell, K. A. Peterson, E. M. Thomas, K. A. O'Hara, E. Lim and M. L. Chabinyc, Sci. Adv., 2017, 3, e1700434.

50 D. Kim and I. V. Zozoulenko, J. Phys. Chem. B, 2019, 123, 5160-5167. 